\title{
Associations between the expression of MTA1 and VEGF-C in esophageal squamous cell carcinoma with lymph angiogenesis and lymph node metastasis
}

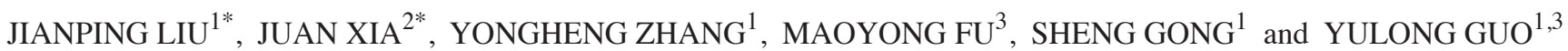 \\ Departments of ${ }^{1}$ Cardiothoracic Surgery and ${ }^{2}$ Pathology, Suining Central Hospital, Suining, Sichuan 629000, \\ ${ }^{3}$ Department of Cardiothoracic Surgery, The Affiliated Hospital of North Sichuan Medical College, \\ Nanchong, Sichuan 637000, P.R. China
}

Received October 5, 2015; Accepted March 23, 2017

DOI: $10.3892 / 01.2017 .6530$

\begin{abstract}
The aim of the present study was to investigate the association between the expression levels of metastasis-related gene 1 (MTA1) and vascular endothelial growth factor C (VEGF-C) in esophageal squamous cell carcinoma (ESCC) with lymph angiogenesis and lymph node metastasis. The paraffin-embedded tissue samples of 107 cases of ESCC and 56 cases of normal esophageal tissues were collected from the Department of Cardiothoracic Surgery, Suining Central Hospital from March 2013 to January 2014. Immunohistochemical assays were performed to detect the expression levels of MTA1, VEGF-C and D2-40 in ESCC, and the micro-lymphatic vessel density (LVD) was evaluated. Their associations with various clinicopathological parameters were also analyzed. The protein expression levels of MTA1 and VEGF-C in ESCC were significantly higher compared with those in normal esophageal tissues $(\mathrm{P}<0.05)$; the high protein expression levels of MTA1 and VEGF-C in ESCC tissues at various tumor-node-metastasis stages exhibited statistically significant differences, as revealed by the Kruskal-Wallis test $(\mathrm{P}<0.05)$. The protein expression levels of MTA1 and VEGF-C in ESCC exhibited positive correlations (Spearman's $\rho, \mathrm{r}=0.512 ; \mathrm{P}=0.000$ ); the LVD level in the group with high expression of MTA1 and VEGF-C was significantly higher compared with in the low expression group $(\mathrm{P}<0.05)$. The comparison between MTA1 and VEGF-C protein expression levels in the group with a high rate of lymph node metastasis demonstrated statistically significant differences when compared with in the low lymph node metastasis
\end{abstract}

Correspondence to: Professor Yongheng Zhang, Department of Cardiothoracic Surgery, Suining Central Hospital, 27 Dong Ping Street, Suining, Sichuan 629000, P.R. China E-mail: jianpingliudoc@126.com

${ }^{*}$ Contributed equally

Key words: metastasis-related gene 1, vascular endothelial growth factor $\mathrm{C}$, esophageal squamous cell carcinoma, lymph angiogenesis group $(\mathrm{P}<0.05)$. The expression levels of MTA1 and VEGF-C in ESCC exhibited a positive correlation in ESCC, which may co-promote lymph angiogenesis and lymph node metastasis in ESCC; therefore, they may be used as biomarkers for determining the prognosis of ESCC.

\section{Introduction}

Esophageal squamous cell carcinoma (ESCC) is one of the most common gastrointestinal malignancies in China, and its associated tumor invasion and metastasis are important contributing factors towards patient mortality and presents a serious risk to health $(1,2)$. A previous study suggested that ESCC primarily exhibits metastasis via lymphatic vessels in the early stages $(1,2)$. Therefore, investigating the factors that may affect lymph angiogenesis in ESCC, as well as the molecular mechanisms underlying lymphatic metastasis, may have vital clinical significance towards the diagnosis and treatment of ESCC (3). Vascular endothelial growth factor C (VEGF-C) is a member of the vascular endothelial growth factor family, and previous studies have demonstrated that VEGF-C participates in the lymph angiogenesis of a variety of malignant tumors, resulting in the lymph node metastasis of tumors and thus affecting prognosis (4-6). Tumor metastasis-related gene 1 (MTA1) occupies a specific position in the entire tumor metastasis-related gene family. A previous study demonstrated that MTA1 protein is a subunit of the nucleosome remodeling and histone deacetylase NuRD (7). It regulates the state of histone deacetylase (HDAC) and nucleosomes by interacting with HDAC so as to regulate its biological functions. In addition, previous studies have implicated MTA1 in multiple types of cancer, including gastric, colon, breast, non-small cell lung, prostate, ovarian and esophageal cancer, and, head and neck squamous cell carcinoma (8-10). Previous studies have primarily focused on the role of MTA1 in tumor angiogenesis, suggesting that it serves crucial functions in tumor angiogenesis in addition to promoting the invasion and metastasis of tumors, while its roles in lymph angiogenesis remain unclear (7-10). Therefore, the present study used immunohistochemistry to detect MTA1, VEGF-C and carcinoembryonic antigen M2A monoclonal antibody (D2-40) in resected tissue 
samples from patients with ESCC, aiming to investigate the roles of MTA1 and VEGF-C in lymph angiogenesis and lymph node metastasis in ESCC, and the associations between them.

\section{Materials and methods}

Sample sources. The ESCC tissues were surgically resected and collected from 107 patients with ESCC from the Department of Cardiothoracic Surgery, Suining Central Hospital (Suining, China) from March 2013 to January 2014, and 56 samples of normal esophageal tissues were also collected during the same period at the same location. Clinicopathological data for all patients is provided in Table I. All tissue samples were paraffin-embedded for storage prior to further experiments. All patients involved did not receive preoperative radiotherapy, chemotherapy, traditional Chinese medicine treatment or any other type of treatment, and had no family history of the disease. The diagnosis for all patients with ESCC was confirmed by postoperative pathology, and tumor-node-metastasis (TNM) staging and tumor grading were performed based on the ESCC staging criteria of American Joint Committee on Cancer/Union for International Cancer Control 7th edition, 2009 (2). The present study was performed in accordance with the declaration of Helsinki and was approved by the Ethics Committee of Suining Central Hospital. Written informed consent was obtained from all patients, prior to enrollment in the present study.

Experimental methods and immunohistochemical evaluations. The paraffin-embedded tissues were cut into $4 \mu \mathrm{m}$ sections and stored at $4^{\circ} \mathrm{C}$, followed by dewaxing, hydration and closure in $3 \%$ hydrogen peroxide in darkness for $20 \mathrm{~min}$ at $20^{\circ} \mathrm{C}$. The tissue sections were then washed with distilled water for $5 \mathrm{~min}$ three times. Subsequently, antigen retrieval was performed as follows: A plastic section tank, filled with $0.01 \mathrm{~mol} / 1$ citric acid buffer ( $\mathrm{pH} 6.0$ ), was placed into a microwave for 10-min pre-heating (high-middle power), then tissue sections were added and microwave antigen retrieval was performed for $20 \mathrm{~min}$ (high power), $10 \mathrm{~min}$ (middle-high power) prior to cooling to room temperature. Tissue sections were again washed with PBS for 5 min three times. Subsequently, the mouse anti-human tumor MTA1 (A11; no. sc-17773; 1:50 dilution; Santa Cruz Biotechnology, Inc., Dallas, TX, USA), rabbit anti-human VEGF-C (H-190; no. sc-9047; 1:50 dilution; Santa Cruz Biotechnology, Inc.) and mouse anti-human D2-40 immunohistochemical monoclonal antibodies (no. MAB-0567; 1:100 dilution; Fuzhou Maxin Biotech Co., Ltd., Fuzhou, China) were added dropwise, then placed into a wetbox for $1 \mathrm{~h}$ at $37^{\circ} \mathrm{C}$, prior to overnight storage at $4^{\circ} \mathrm{C}$. The sections were then washed with PBS for $5 \mathrm{~min}$ three times. Secondary antibodies (Envision ${ }^{\mathrm{TM}}$ kit and chromogenic agents; Dako REAL ${ }^{\mathrm{TM}}$ EnVision $^{\mathrm{TM}}$ Detection System, Peroxidase/DAB+, Rabbit/Mouse; cat. no. K5007) were then added dropwise, placed into a wetbox for $1 \mathrm{~h}$ culture at $37^{\circ} \mathrm{C}$, prior to washing with PBS for $5 \mathrm{~min}$ three times. The residual PBS on the tissue sections was discarded and DAB solution was added and samples were incubated for $10 \mathrm{sec}$ at room temperature. The tissue sections were placed into distilled water and hematoxylin re-staining was performed for $3 \mathrm{~min}$ at room temperature. Following hydrochloric acid-ethanol differentiation, the tissue sections were washed with running water for $10 \mathrm{~min}$ and dehydrated sequentially using 85,95 and $100 \%$ ethanol for $5 \mathrm{~min}$ at each gradient, and finally mounted with green mounting glue.

PBS was used to replace the primary antibodies for the control tissues and breast tissues with clear expression were used as the positive control (11). Two experienced pathologists who were blinded to the identity of each sample conducted the determination of immunohistochemical results independently. The tissue sections were scored according to the proportion of positive cells: $0, \leq 5 \% ; 1,6-25 \% ; 2,26-50 \% ; 3,51-75 \%$; $4,76-100 \%$. The staining intensity was scored as follows: 0 , no signal; 1 , pale yellow; 2 , buffy; 3 , brownish. Comprehensive evaluation: The results were then scored based on the product of the positive cell proportion and the staining intensity: 0 , 0-1 score; 1, 2-4 score; 2, 6-8 score; 3, 9-12 score; high expression, $\geq 2$ points; low expression, $<2$ points $(12,13)$. LVD in ESCC tissue sections that were single-stained for D2-40 was analyzed as previously described. Tumoral LVD located at the periphery of tissue, within $2 \mathrm{~mm}$ of the tumor and adjacent to the invasive front was assessed. Five areas where the most lymphatic vessels could be observed were chosen using light microscopy at x40 magnification. LVD was assessed by counting all the stained vessels in each area at x200 magnification. The assessed mean number of lymphatic vessels was determined and expressed as LVD (14).

Statistical methods. SPSS version 13.0 (SPSS, Inc., Chicago, IL, USA) was used to analyze the experimental data. The $\chi^{2}$ test was used to analyze the differences between high and low expression levels of MTA1 and VEFG-C, and Kruskal-Wallis one-way analysis of variance was used to analyze the measurement data among $>3$ groups. The correlations between MTA1 and VEGF-C expression levels were analyzed using Spearman's $\rho$ correlation analysis. Comparison of the means between the groups was performed using a Student's t-test. $\mathrm{P}<0.05$ was considered to indicate a statistically significant difference.

\section{Results}

Characteristics of positive immunohistochemical expression. Immunohistochemical analysis results suggested that the positive signal of MTA1 protein was located in the nuclei of ESCC cells (Fig. 1) and the positive signal of VEGF-C protein was located in the cytoplasm of ESCC cells (Fig. 2). D2-40 staining was mainly located in the tumor stroma microenvironment surrounding the ESCC tumor tissues (Fig. 3).

\section{Expression levels of MTA1 and VEGF-C and its correlations with the clinicopathological parameters in ESCC tissues. In ESCC, the protein expression level of MTA1 was 50.4\% and that of VEGF-C was $58.8 \%$, which are significantly high compared with those in the normal esophageal tissues $(\mathrm{P}<0.05)$. Among the $107 \mathrm{ESCC}$ tissue samples, the expression levels of MTA1 and VEGF-C proteins in the group with lymph node metastasis were 58.7 and $68.3 \%$, respectively, and those in the group without lymph node metastasis were 38.6 and $45.3 \%$, respectively, differences that were statistically signifi- cant $(\mathrm{P}<0.05)$. In the $\mathrm{T} 3 / 4$ group, the expression level of MTA1 and VEGF-C proteins was 58.6 and $65.7 \%$, respectively, and}


Table I. Clinicopathological data of the 107 patients with ESCC.

\begin{tabular}{lc}
\hline Clinicopathological data & $\begin{array}{c}\text { Distribution } \\
\text { of patients }\end{array}$ \\
\hline Age (mean \pm standard deviation) & $61.15 \pm 7.12$ \\
Sex (male:female) & $86: 21$ \\
Lesion length ( $\geq 5 /<5 \mathrm{~cm})$ & $35 / 72$ \\
Tumor type (visual assessment) & $76 / 13 / 10 / 8$ \\
(ulcerative/fungating/medullary/rosive) & \\
Differentiation degree (high/medium/low) & $24 / 65 / 18$ \\
TNM staging (I/II/III) & $10 / 50 / 47$ \\
T stage (T1/T2/T3/T4) & $12 / 25 / 64 / 6$ \\
N stage (N0/N1/N3/N) & $44 / 39 / 19 / 5$ \\
\hline
\end{tabular}

ESCC, esophageal squamous cell carcinoma; TNM, tumor-nodemetastasis.

in the T1/2 group it was 35.1 and $45.9 \%$, respectively, differences that were also statistically significant $(\mathrm{P}<0.05)$. There was no correlation between high expression levels of MTA1 and VEGF-C proteins and the patient age, sex, differentiation degree or tumor size $(\mathrm{P}>0.05$; Table II).

Associations between the expression levels of MTA1 and $V E G F-C$ and patient clinical staging and lymph vessel density in ESCC. The high expression levels of MTA1 and VEGF-C proteins in various TNM stage ESCC tissue samples were compared using Kruskal-Wallis one-way analysis of variance, and the differences were statistically significant $(\mathrm{P}<0.05$; Table III). In the group with high expression levels of MTA1, the micro-lymphatic density (LVD) was $15.784 \pm 3.874 /$ high-power field (HPF), which was significantly different compared with in the group with low MTA1 expression levels $(11.550 \pm 3.341 / \mathrm{HPF}$; $\mathrm{P}<0.05)$. In the group with a high expression level of VEGF-C, LVD was $15.333 \pm 3.803 / \mathrm{HPF}$, which was significantly different compared with a low expression level of VEGF-C in the group with low LVD (11.333 $\pm 3.556 / \mathrm{HPF} ; \mathrm{P}<0.05)$.

Association between the protein expression levels of MTAI and VEGF-C in ESCC. The protein expression levels of MTA1 and VEGF-C in ESCC were determined using Spearman's rank correlation test. The results revealed that they were positively correlated $(\mathrm{r}=0.512 ; \mathrm{P}<0.000$; Table IV).

\section{Discussion}

Cases of esophageal cancer in China are typically ESCC, which is the fifth most common type of cancer in China and has an associated mortality rate ranked as the fourth highest among malignant types of cancer (3). ESCC may be spread in vivo via the direct invasion of surrounding tissues, including lymphatic metastasis, hematogenous metastasis and implantation metastasis (2). Invasion and metastasis remain important factors that affect the post-operative 5-year survival rate of patients with ESCC $(1,2)$. The esophagus lacks the serosal layer and is rich in lymphatic drainage; therefore, ESCC may be transferred mainly via lymphatic metastasis in the early stages. Furthermore, investigating the factors that affect lymph angiogenesis in ESCC, as well as the molecular mechanisms underlying lymphatic metastasis, may have important clinical value for the diagnosis and treatment $\operatorname{ESCC}(15,16)$.

It has previously been demonstrated that D2-40 was a lymphatic endothelial cell marker with high specificity, thus the present study used D2-40 to mark the lymphatic endothelial cells in ESCC and normal esophageal tissues, and to determine the $\operatorname{LVD}(17,18)$. The present study revealed that LVD was high in the tumor microenvironment surrounding ESCC, and were irregular in shape and exhibited the extended state. Whereas LVD was low in the central region of ESCC, mostly with strip-like shapes and exhibiting the blocking state, with only a few exhibiting the extended state, similar to a previous study by Padera et al (19). These structural characteristics may allow micro-lymphatic vessels to become the direct channel for the invasion and metastasis of malignant tumors. In the present study, the LVD in the ESCC group (13.688 \pm 4.183$)$ was significantly different compared with that in the normal esophageal group (9.165 $\pm 2.284 ; \mathrm{P}<0.05)$. LVD in the surrounding area (stroma) of ESCC was significantly higher compared with those in the central region of ESCC and normal esophageal tissues; the comparison of LVD between the 44 cases without lymph node metastasis $(12.474 \pm 4.647)$ and 63 cases with lymph node metastasis $(14.676 \pm 3.473)$, revealed that LVD in the group with lymph node metastasis was significantly higher compared with in tissues without lymph node metastasis, and the difference was statistically significant $(\mathrm{P}<0.05)$. Therefore, LVD in ESCC was increased compared with control tissue, and LVD was associated with lymph node metastasis.

MTA1 is a novel tumor metastasis-associated gene, highly expressed in normal human testis, with no or low expression in other non-cancerous tissues; it may be upregulated to various degrees in numerous types of cancer and is closely associated with tumor metastasis and invasion $(11,20)$. However, these previous studies were limited to the investigation of associations between MTA1 and prognosis, or between MTA1 and tumor angiogenesis, and did not investigate the associations between MTA1 and tumor lymph angiogenesis. The present study demonstrated that the expression level of MTA1 protein in ESCC was significantly higher compared with in normal esophageal tissues $(\mathrm{P}<0.05)$. MTA1 protein was primarily expressed in the nuclei of ESCC cells, and with increasing ESCC stage, the protein expression levels of MTA1 gradually increased. Among the 63 patients with ESCC lymph node metastasis, 37 exhibited high expression levels of MTA1 protein. Among the 44 patients with ESCC without lymph node metastasis, 17 exhibited high expression levels of MTA1 protein. For each group, the difference was statistically significant $(\mathrm{P}<0.05)$. In ESCC, LVD in the group with high expression of MTA1 was significantly high compared with in the group with low MTA1 expression $(\mathrm{P}<0.05)$, which indicated that the MTA1 gene may serve a promoting role in the development, lymph angiogenesis and lymph node metastasis of ESCC.

Previous studies have revealed that VEGF-C is overexpressed in numerous types of human malignancies, and participates in the lymph angiogenesis of certain malignant types of cancer, resulting in the progression and lymph node metastasis of primary tumors and ultimately affecting patient 


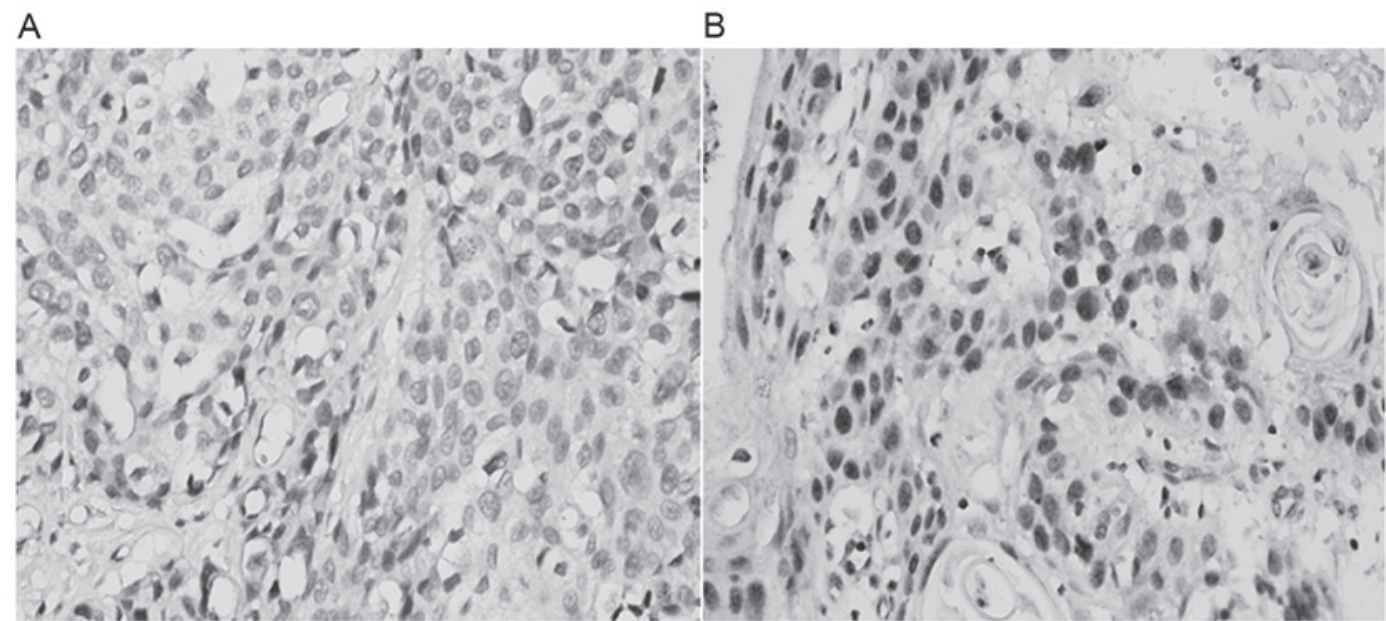

Figure 1. Expression level of MTA-1 in ESCC. The positive signals were primarily located in the nuclei of the ESCC cells. (A) Low expression level of MTA-1 in ESCC (magnification, x200). (B) High expression level of MTA-1 in ESCC (magnification, x200). MTA-1, metastasis-related gene 1; ESCC, esophageal squamous cell carcinoma.

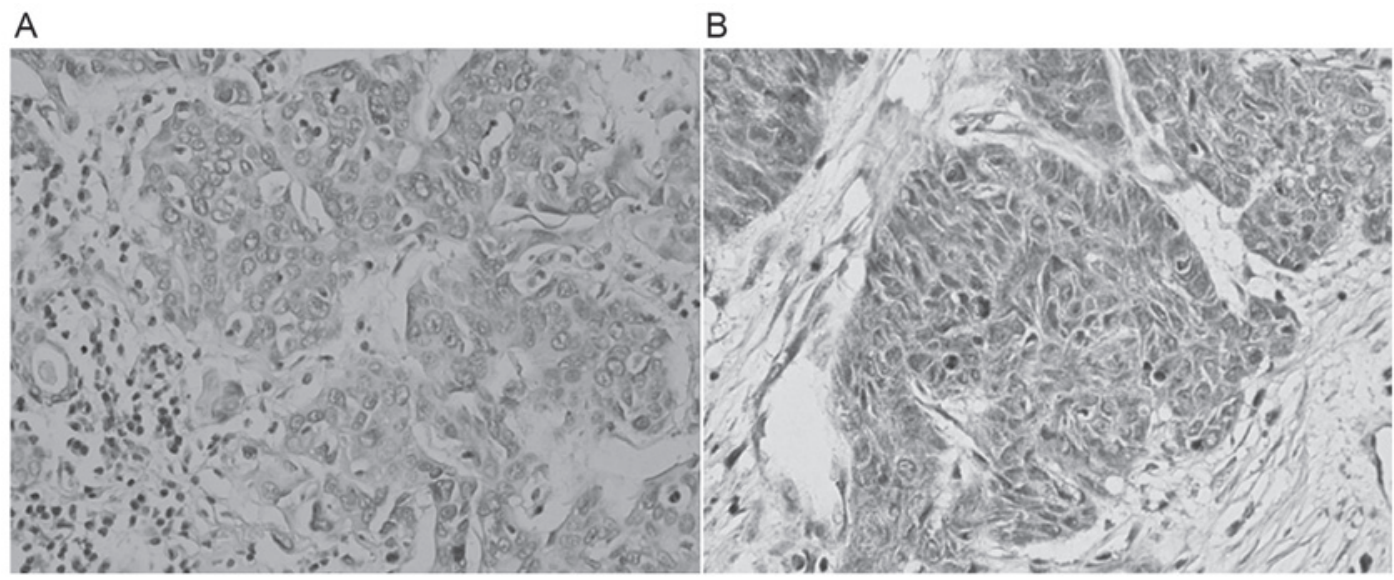

Figure 2. Expression levels of VEGF-C in ESCC. The positive signals were principally located in the cytoplasm of the ESCC cells. (A) Low expression levels of VEGF-C in ESCC (magnification, x200). (B) High expression levels of VEGF-C in ESCC (magnification, x200). ESCC, esophageal squamous cell carcinoma; VEGF-C, vascular endothelial growth factor $\mathrm{C}$.

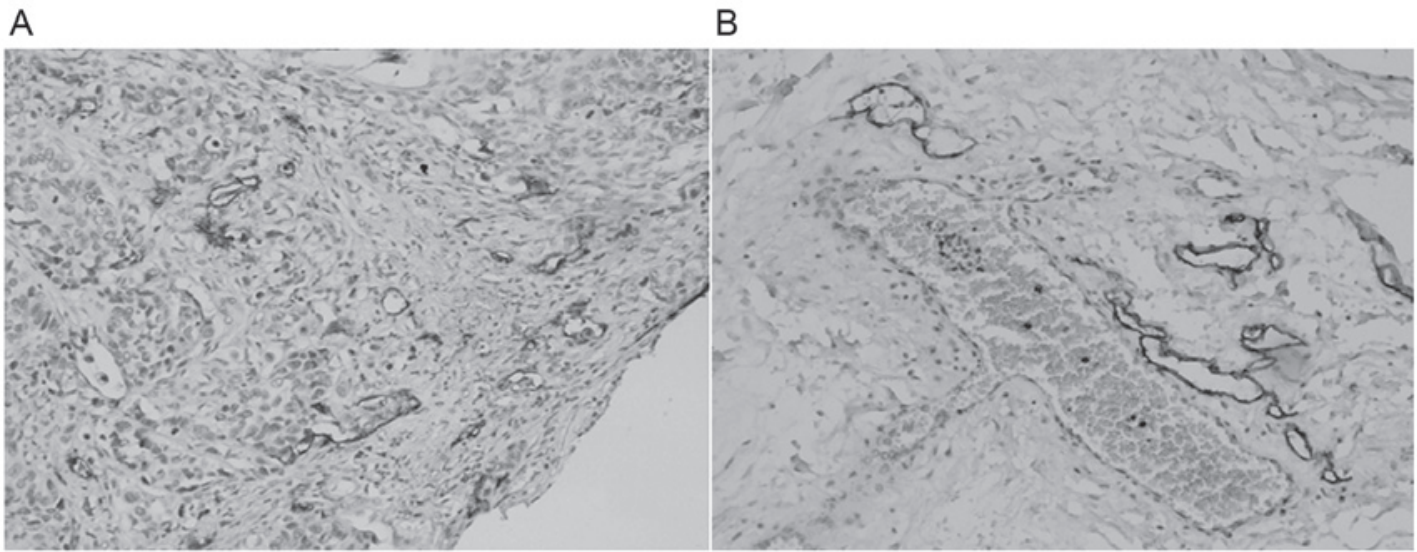

Figure 3. Expression levels of D2-40 in ESCC. (A) Positively stained lymphatic vessels were mainly located in the tumor stroma surrounding the ESCC tumor tissues and had more irregular morphology compared with normal esophageal tissue (magnification, x100). (B) Lymphatic D2-40 was stained positively, but the adjacent venous wall was negative for D2-40 expression (magnification, x200). ESCC, esophageal squamous cell carcinoma.

prognosis (21-23). The results of the present study suggested that VEGF-C protein was predominantly expressed in the cytoplasm of ESCC cells, and with increasing tumor stage the protein expression levels of VEGF-C were also increased, 


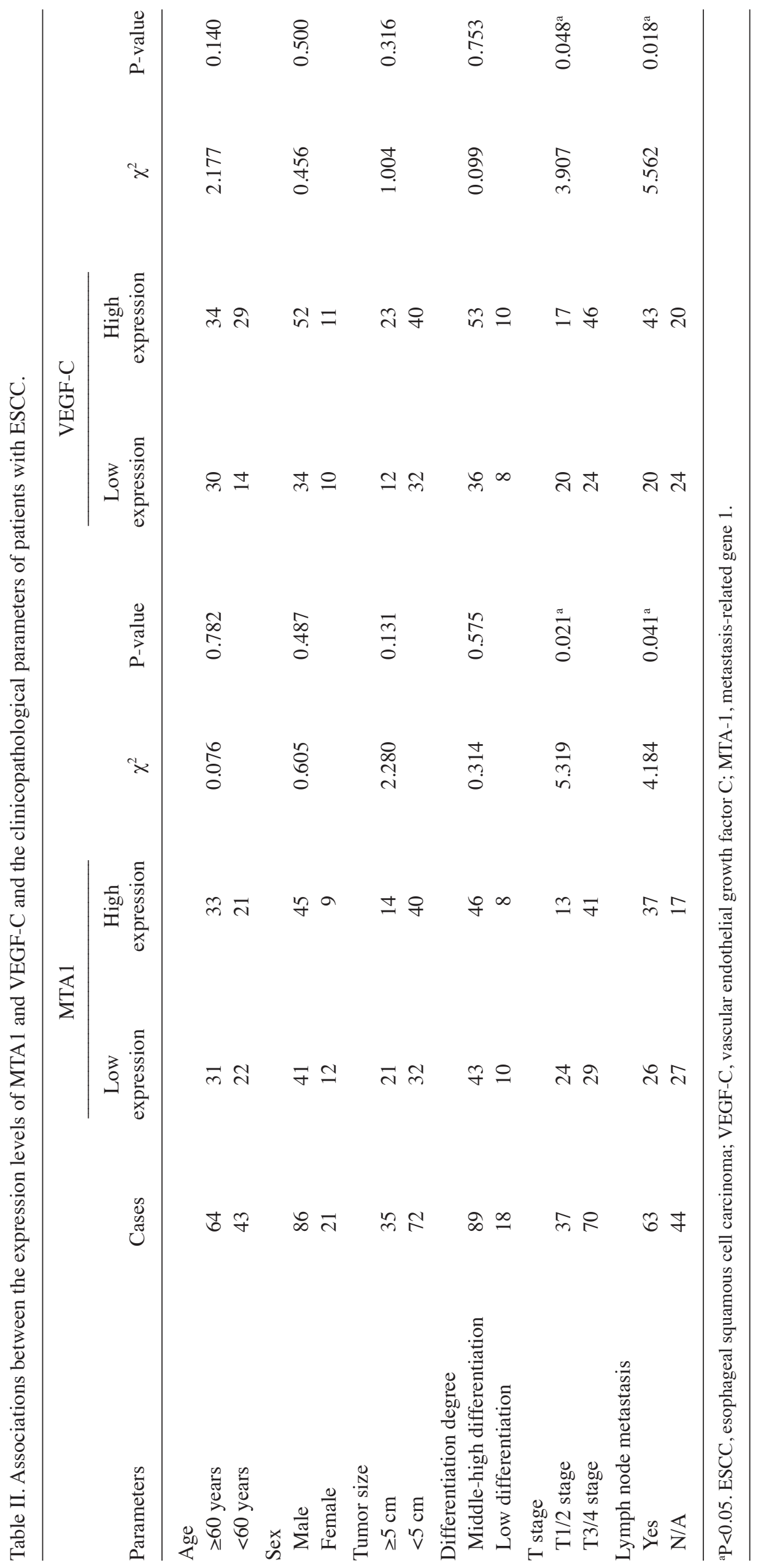


Table III. Associations between the expression levels of MTA1 and VEGF-C with clinical staging of ESCC.

\begin{tabular}{|c|c|c|c|c|c|c|c|c|}
\hline \multirow[b]{2}{*}{ Stage } & \multicolumn{3}{|c|}{ MTA1 } & \multirow[b]{2}{*}{$\chi^{2}$} & \multicolumn{3}{|c|}{ VEGF-C } & \multirow[b]{2}{*}{$\chi^{2}$} \\
\hline & $\begin{array}{c}\text { Low } \\
\text { expression }\end{array}$ & $\begin{array}{c}\text { High } \\
\text { expression }\end{array}$ & Sum & & $\begin{array}{c}\text { Low } \\
\text { expression }\end{array}$ & $\begin{array}{c}\text { High } \\
\text { expression }\end{array}$ & Sum & \\
\hline I & 7 & 3 & 10 & 6.407 & 7 & 3 & 10 & 6.058 \\
\hline II & 29 & 21 & 50 & $\mathrm{P}=0.041$ & 22 & 28 & 50 & $\mathrm{P}=0.048$ \\
\hline III & 17 & 30 & 47 & & 14 & 33 & 47 & \\
\hline Sum & 53 & 54 & 107 & & 44 & 63 & 107 & \\
\hline
\end{tabular}

ESCC, esophageal squamous cell carcinoma; VEGF-C, vascular endothelial growth factor C; MTA-1, metastasis-related gene 1.

Table IV. Associations between the protein expression levels of MTA1 and VEGF-C in ESCC.

\begin{tabular}{lrrrrr}
\hline & \multicolumn{4}{c}{ VEGF-C } \\
\cline { 2 - 4 } MTA1 & 0 point & 1 point & 2 points & 3 points & Sum \\
\hline 0 point & 10 & 5 & 4 & 2 & 21 \\
1 point & 3 & 13 & 13 & 3 & 32 \\
2 points & 1 & 7 & 15 & 4 & 27 \\
3 points & 0 & 5 & 6 & 16 & 27 \\
Sum & 14 & 30 & 38 & 25 & 107 \\
\hline
\end{tabular}

ESCC, esophageal squamous cell carcinoma; VEGF-C, vascular endothelial growth factor C; MTA-1, metastasis-related gene 1 . The correlation coefficient of MTA1 and VEGF-C in ESCC; $(r=0.512$; $\mathrm{P}<0.000)$.

suggesting that the VEGF-C gene may serve a specific promoting role in the development of ESCC. The protein expression level of VEGF-C in ESCC was significantly high, compared with in the normal esophageal tissues $(\mathrm{P}<0.05)$. Peng et al (24) retrieved the VEGF-associated literature in PubMed and Embase databases and performed a meta-analysis of 19 studies that met the final conditions (including 1,453 patient cases), and concluded that the overexpression of VEGF-C was positively associated with the poor prognosis of ESCC. In particular, Asian patients with ESCC and positive VEGF-C expression exhibited a poorer prognosis when compared with those patients with negative VEGF-C expression level (23). VEGF-C was an effective indicator of the prognosis of ESCC; however, prospective studies are required for further confirmation (24). In the present study, among the 63 patients with ESSC and lymph node metastasis, 43 exhibited a high protein expression level of VEGF-C, and among the 44 ESSC patients without lymph node metastasis, 20 had high protein expression levels of VEGF-C, a difference that was statistically significant $(\mathrm{P}<0.05)$. In ESCC tissues, LVD in the group with a high VEGF-C expression was significantly higher compared with in the group with low VEGF-C expression $(\mathrm{P}<0.05)$. Therefore, the present study hypothesized that detecting the expression level of VEGF-C in biopsy specimens may aid the prediction of lymph node metastasis in ESCC.
Table V. TNM staging of esophageal squamous cell carcinoma and other non adenocarcinomas (AJCC/UICC, 2009), seventh edition.

\begin{tabular}{llllll}
\hline TNM & T & N & M & G & \multicolumn{1}{c}{ Segment } \\
\hline 0 & Tis & N0 & M0 & G1,X & Any \\
IA & T1 & N0 & M0 & G1,X & Any \\
IB & T1 & N0 & M0 & G2-3 & Any \\
& T2-3 & N0 & M0 & G1,X & Lower, X \\
IIA & T2-3 & N0 & M0 & G1,X & Middle, Upper \\
& T2-3 & N0 & M0 & G2-3 & Lower, X \\
IIB & T2-3 & N0 & M0 & G2-3 & Middle, Upper \\
& T1-2 & N1 & M0 & Any & Any \\
IIIA & T1-2 & N2 & M0 & Any & Any \\
& T3 & N1 & M0 & Any & Any \\
& T4a & N0 & M0 & Any & Any \\
IIIB & T3 & N2 & M0 & Any & Any \\
IIIC & T4a & N1-2 & M0 & Any & Any \\
& T4b & Any & M0 & Any & Any \\
& Any & N3 & M0 & Any & Any \\
IV & Any & Any & M1 & Any & Any \\
\hline
\end{tabular}

Moon et al (25) revealed that MTA1 increased the expression and stability of hypoxia-inducible factor $1-\alpha(\mathrm{HIF}-1 \alpha)$, and that HIF- $1 \alpha$ also promoted tumor angiogenesis by regulating VEGF; a high expression level of MTA1 was also indicated to increase the metastatic potential of tumor cells (26). To the best of our knowledge, no previous studies have investigated the association between MTA1 and VEGF-C expression in ESCC, in addition to their association with tumor lymph angiogenesis. Using the $\chi^{2}$ test, the present study revealed significant differences between the expression levels of MTA1 and VEGF-C present in the ESCC cases with lymph node metastasis (37/63, 43/63) and those in the ESCC cases without lymph node metastasis (17/44, 20/44; $\mathrm{P}=0.041, \mathrm{P}=0.018$, respectively). In addition, LVD in the ESCC cases with high expression levels of MTA1 and VEGF-C was significantly higher compared with in the ESCC cases with low expression levels of MTA1 and VEGF-C $(\mathrm{P}<0.05)$. This suggested that the expression levels of MTA1 and VEGF-C may be closely associated with lymph 
angiogenesis in ESCC, and that with increasing expression levels the likelihood of lymph node metastasis in ESCC may be correspondingly increased. The Spearman's $\rho$ test revealed that the protein expression levels of MTA1 and VEGF-C were positively associated in ESCC $(r=0.512 ; \mathrm{P}<0.000)$, indicating that MTA1 and VEGF-C may serve synergic roles in the lymph angiogenesis of ESCC, which could co-promote the growth and lymphatic metastasis of ESCC. Therefore, according a study performed by Moon et al (25), the present study hypothesized that MTA1 may adhere to HIF-1 $\alpha$, thereby regulating the expression of VEGF-C and promoting lymph angiogenesis in ESCC; however, the specific underlying regulatory mechanisms require further experimental study.

\section{References}

1. Lin CS, Cheng CT, Liu CY, Lee MY, Hsiao MC, Shih CH and Liu CC: Radical lymph node dissection in primary esophagectomy for esophageal squamous cell carcinoma. Ann Thorac Surg 100: 278-286, 2015.

2. Edge SB, Byrd DR, Compton CC, Fritz AG, Greene FL and Trotti A (eds): AJCC Cancer Staging Handbook. 7th edition. Springer, New York, NY, 2010.

3. Xu XL, Zheng WH, Zhu SM, Zhao A and Mao WM: The prognostic impact of lymph node involvement in large scale operable node-positive esophageal squamous cell carcinoma patients: A 10-year experience. PLoS One 10: e0133076, 2015.

4. Feng Y, Wang W, Hu J, Ma J, Zhang Y and Zhang J: Expression of VEGF-C and VEGF-D as significant markers for assessment of lymphangiogenesis and lymph node metastasis in non-small cell lung cancer. Anat Rec (Hoboken) 293: 802-812, 2010.

5. Wakisaka N, Hirota K, Kondo S, Sawada-Kitamura S, Endo K, Murono $\mathrm{S}$ and Yoshizaki T: Induction of lymphangiogenesis through vascular endothelial growth factor-C/vascular endothelial growth factor receptor 3 axis and its correlation with lymph node metastasis in nasopharyngeal carcinoma. Oral Oncol 48: 703-708, 2012.

6. Pérez D, Rohde A, Callejón G, Pérez-Ruiz E, Rodrigo I, Rivas-Ruiz F, Ramos B, Medina F, Villatoro R, Redondo M, et al Correlation between serum levels of vascular endothelial growth factor-C and sentinel lymph node status in early breast cancer. Tumour Biol 36: 9285-9293, 2015.

7. Li DQ, Suresh SB, Sujit SS, Eswaran J and Kumar R: Metastasis-associated protein 1/nucleosome remodeling and histone deacetylase complex in cancer. Cancer Res 72: 387-394, 2012.

8. Li SH, Tian H, Yue WM, Li L, Li WJ, Chen ZT, Hu WS, Zhu YC and Qi L: Overexpression of metastasis-associated protein 1 is significantly correlated with tumor angiogenesis and poor survival in patients with early-stage non-small cell lung cancer. Ann Surg Oncol 18: 2048-2056, 2011.

9. Kai L, Wang J, Ivanovic M, Chung YT, Laskin WB, SchulzeHoepfner F, Mirochnik Y, Satcher RL Jr and Levenson AS: Targeting prostate cancer angiogenesis through metastasis-associated protein 1 (MTA1). Prostate 71: 268-280, 2011.

10. Prisco MG, Zannoni GF, De Stefano I, Vellone VG, Tortorella L, Fagotti A, Mereu L, Scambia G and Gallo D: Prognostic role of metastasis tumor antigen 1 in patients with ovarian cancer: A clinical study. Hum Pathol 43: 282-288, 2012.

11. Nagaraj SR, Shilpa P, Rachaiah K and Salimath BP: Crosstalk between VEGF and MTA1 signaling pathway to aggressiveness of breast carcinoma. Mol Carcinog 54: 333-350, 2015.
12. Hofer MD, Kuefer R, Varambally S, Li H, Ma J, Shapiro GI, Gschwend JE, Hautmann RE, Sanda MG, Giehl K, et al: The role of metastasis-associated protein 1 in prostate cancer progression. Cancer Res 64: 825-829, 2004

13. Miyake K, Yoshizumi T, Imura S, Sugimoto K, Batmunkh E, Kanemura H, Morine Y and Shimada M: Expression of hypoxiainducible actor-1alpha, histone deacetylase 1, and metastasis-associated protein 1 in pancreatic carcinoma: Correlation with poor prognosis with possible regulation. Pancreas 36: e1-e9, 2008.

14. Aishima S, Nishihara Y, Iguchi T, Taguchi K, Taketomi A, Maehara Y and Tsuneyoshi M. Lymphatic spread is related to VEGF-C expression and D2-40-positive myofibroblasts in intrahepatic cholangiocarcinoma. Mod Pathol 21: 256-264, 2008.

15. Sawyers CL: The cancer biomarker problem. Nature 452: 548-552, 2008

16. Zamanian-Azodi M, Rezaei-Tavirani M,Hasanzadeh H, Rahmati Rad S and Dalilan S: Introducing biomarker panel in esophageal, gastric, and colon cancers; A proteomic approach. Gastroenterol Hepatol Bed Bench 8: 6-18, 2015.

17. Saad RS, Lindner JL, Liu Y and Silverman JF: Lymphatic vessel density as prognostic marker in esophageal adenocarcinoma. Am J Clin Pathol 131: 92-98, 2009.

18. Straume O, Jackson DG and Akslen LA: Independent prognostic impact lymphatic vessel density and presence of low-grade lymphangiogenesis in cutaneous melanoma. Clin Cancer Res 9: 250-256, 2003.

19. Padera TP, Kadambi A, di Tomaso E, Carreira CM, Brown EB, Boucher Y, Choi NC, Mathisen D, Wain J, Mark EJ, et al: Lymphatic metastasis in the absence of functional intratumor lymphatics. Science 296: 1883-1886, 2002.

20. Deng X, Du L, Wang C, Yang Y, Li J, Liu H, Zhang J, Wang L, Zhang X, Li W, et al: Close association of metastasis-associated protein 1 overexpression with increased angiogenesis and poor survival in patients with histologically node-negative gastric cancer. World J Surg 37: 792-798, 2013.

21. Xie LX, Zhai TT, Yang LP, Yang E, Zhang XH, Chen JY and Zhang $\mathrm{H}$ : Lymphangiogenesis and prognostic significance of vascular endothelial growth factor $\mathrm{C}$ in gastro-oesophageal junction adenocarcinoma. Int J Exp Pathol 94: 39-46, 2013.

22. Ochi N, Matsuo Y, Sawai H, Yasuda A, Takahashi H, Sato M, Funahashi H, Okada Y and Manabe T: Vascular endothelial growth factor-C secreted by pancreatic cancer cell line promotes lymphatic endothelial cell migration in an in vitro model of tumor lymphangiogenesis. Pancreas 34: 444-451, 2007.

23. Wang CA, Harrell JC, Iwanaga R, Jedlicka P and Ford HL: Vascular endothelial growth factor $\mathrm{C}$ promotes breast cancer progression via a novel antioxidant mechanism that involves regulation of superoxide dismutase 3. Breast Cancer Res 16: 462, 2014.

24. Peng J, Shao N, Peng H and Chen LQ: Prognostic significance of vascular endothelial growth factor expession in esophageal carcinoma: A meta-analysis. J BUON 18: 398-406, 2013.

25. Moon HE, Cheon H, Chun KH, Lee SK, Kim YS, Jung BK, Park JA, Kim SH, Jeong JW and Lee MS: Metastasis-associated protein 1 enhances angiogenesis by stabilization of HIF-1 $\alpha$. Oncol Rep 16: 929-935, 2006.

26. Du B, Yang ZY, Zhong XY, Fang M, Yan YR, Qi GL, Pan YL and Zhou XL: Metastasis-associated protein 1 induces VEGF-C and facilitates lymphangiogenesis in colorectal cancer. World J Gastroenterol 17: 1219-1226, 2011. 